\title{
An Adaptive System for Underwater Wireless Power Transfer
}

\author{
Francisco Gonçalves, Adriano Pereira, André Morais, Cândido Duarte, Rui Gomes, and L. M. Pessoa \\ INESC TEC and Faculty of Engineering, University of Porto \\ Porto, Portugal \\ Email: candidoduarte@fe.up.pt
}

\begin{abstract}
In wireless power transfer systems, if the driver is not capable of dynamically adapt its own switching frequency, small environmental changes or even slight deviations in circuit parameters may prevent the complete system from working properly when the optimal resonance frequency moves towards new values. In this paper, we propose an adaptive system suitable for underwater wireless applications in sea water. The output voltage is regulated using the wireless power link, avoiding the need for additional wireless interfaces. Our complete system includes the power driver, coupling coils, rectifier, and two micro-controllers. The regulation is accomplished by digital load modulation, observable at the input by means of current sensing at the power supply. Experimental results demonstrate a class-D driver with a series-series resonant topology working in saline water, delivering power between 1.6 and $2.4 \mathrm{~W}$. The regulated voltage is $7.5 \mathrm{~V}$ with error less than $7.2 \%$ in the load range of 30 to $37 \Omega$ and 6 to $10 \mathrm{~V}$ power supply variation. The switching frequency is adjusted within the range of $7 \mathrm{kHz}$ deviation $(-7 \%)$.
\end{abstract}

\section{INTRODUCTION}

Recently, wireless power transfer (WPT) based on resonance has met a wide range of application scenarios [1]. WPT has been addressed in wireless battery charging of smartphones [2], medical implants [3], and electrical vehicles [4], [5]. Such systems rely on non-radiative short-range power transfer, normally comprising one or more pairs of magnetically coupled coils. These operate in resonance to allow a higher energy power transfer, being usually modelled as transformers. Fig. 1 depicts a block diagram for a conventional WPT system. A driver is employed at the primary side, which can be implemented using different techniques, such as classD inverters or class-E drivers. At the secondary side, a halfor full-bridge rectifiers with diodes can be employed to obtain a dc voltage output, often requiring some sort of additional adjustment mechanism. Voltage regulation is usually provided to support load variations and it is typically implemented following the rectifier.

Resonant WPT systems are generally subject to several parameter variations, e.g. due to time-varying distance between coupling coils, unpredictable load variation demands, or any other mechanical or electrical uncertainties that may affect resonance [6]. This is particularly severe in circuits with high loaded quality factors, i.e. where power can solely be transferred across a narrow frequency bandwidth and the WPT system has almost no tunability capabilities at all. To overcome component variations and still provide a constant

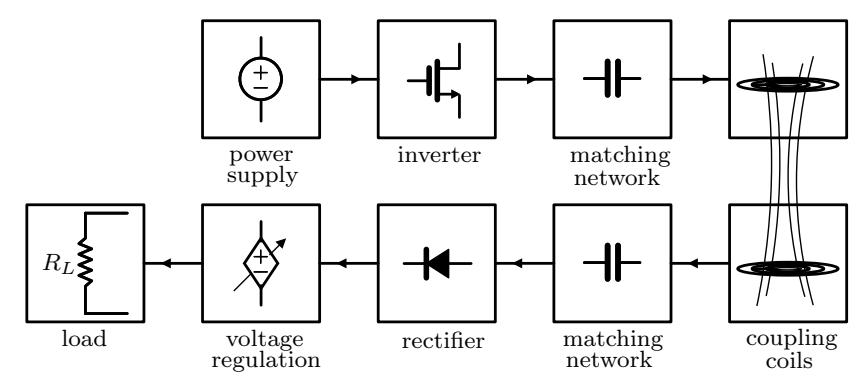

Fig. 1. WPT system block diagram.

output voltage, the authors in [7] make use of a magnetic amplifier to tune the inductance in an LCL pickup circuit. In [8], a reconfigurable four-coil WPT system is presented in which maximum efficiency is systematically tracked by sensing voltage and current to adjust the driver switching frequency. Adaptive approaches need to be adopted to circumvent possible parameter deviations in practical implementations, which may prevent the system from proper operation when resonance or other parameters are slightly different from expected. However, performing parameter tuning in the complete system requires interchanging information between the power transmitter and load circuitry. Recently reported works [9], [10] rely on wireless communications to exchange data for WPT closed-loop optimization control. Unfortunately, such additional wireless data interfaces are not simple to provide in some cases, specially in underwater applications in which operation at high frequencies imply intolerable energy losses. The present paper addresses the power regulation of a dc output voltage with an adaptive approach to look up for the optimum frequency to achieve the desired target voltage. The proposed system aims at providing a wireless power link in sea water with output voltage regulation established without additional links. The next section addresses the proposed system, followed by a section that presents measurement results from the practical implementation.

\section{Proposed Underwater WPT System}

The proposed wireless system intends to power devices in the deep sea for monitoring purposes. To prevent excessive losses due to the conductivity of salt water [11], low frequency is adopted for operation, i.e. around $100 \mathrm{kHz}$. The series-series driver topology is preferred due to its simplicity and to ease 


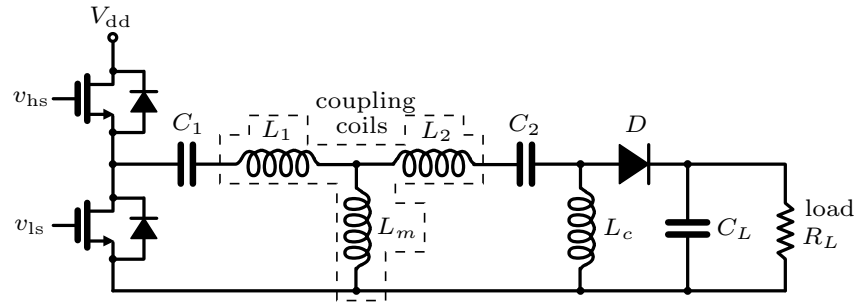

(a)

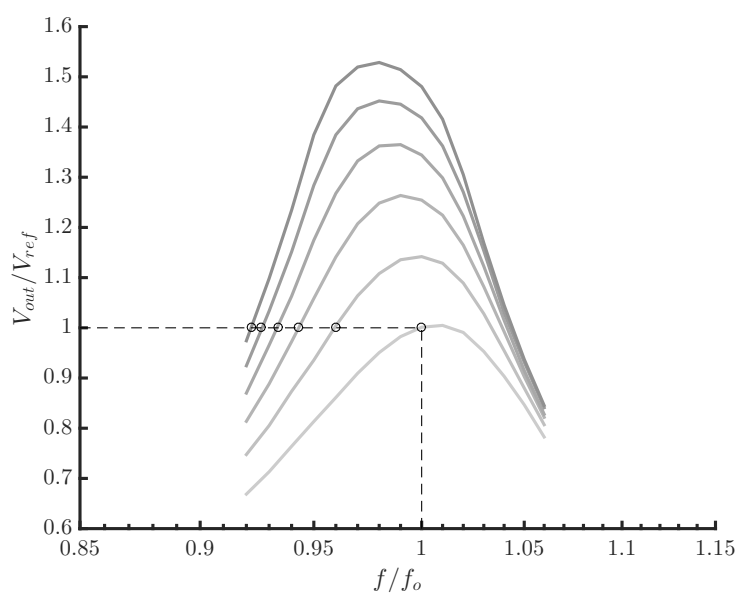

(b)

Fig. 2. (a) Series-series resonant driver topology with rectifier and (b) its typical frequency response.

a stable voltage at the secondary side [5]. Fig. 2(a) depicts a conventional series-series class-D resonant driver. Signals $v_{\mathrm{hs}}$ and $v_{\mathrm{ls}}$ represent respectively the driving signals of the high- and low-side power MOSFETs. The coupling coils are represented as a "tee" model in which the auto inductances are $L_{1}=L_{2}=(1-k) \cdot L$, and the mutual inductance is $L_{m}=k \cdot L$, with $k$ as the coupling factor. The capacitors $C_{1}$ and $C_{2}$ are usually equal valued, and define the resonance frequency. Fig. 2(b) shows the frequency response of the series-series resonant system with a rectifier, i.e. it represents the output dc voltage $V_{\text {out }}$ vs target voltage $V_{\text {ref }}$ if the switching frequency of the driver is statically swept. Each line represents a different load (the load increases monotonically towards the lines to the top), having a peak relatively close to the resonance frequency $f_{o}$.

A voltage regulation algorithm based on hill climbing, using the frequency as the control variable, is proposed to regulate the voltage at the secondary side. As such, we incrementally change the frequency within a certain range preferably in the left side of $f_{o}$, i.e. $f \in\left[f_{\min } ; f_{o}\right]$ as exemplified by the circles represented in Fig. 2(b). This implies a load range in which the regulated voltage at $R_{L, \min }$ occurs at nearly $f_{o}$, although additional limitations may arise in the practical implementation. This optimization can compensate for deviations of the resonance frequency in a given range. However, to change the frequency, the driver has to be able to determine the output voltage, whether it is within an acceptable regulation tolerance or not. The input current can be used to



Fig. 3. Load modulation signals for regulating $V_{\text {out }}$.

optimize the operation of the driver [12]. To do so, we propose load modulation in which the output voltage is compared to a reference $\left(V_{\text {ref }}\right)$, and takes two different actions when the voltage is above or below a certain tolerance. As such, we provide periodic short-circuits to the load for a predefined time so that, at the primary side, abrupt changes in the current drawn from the power source $V_{\mathrm{dd}}$ can be sensed. Two fixedtime pulses (duration at "high" state of $50 \mathrm{~ms}$ ) are generated, spaced by 100 and $150 \mathrm{~ms}$ in case of $V_{\text {out }}$ being above and below, respectively. Following the two pulses, the sensing is disabled for 2 sec., so that the driver can update the switching frequency (see Fig. 3).

In a series-series driver topology, the higher the load, the higher the voltage (and current). This behavior implies that for the load modulation, short circuits are preferred to avoid increasing the power consumption for modulation purposes. On the primary side, the power-supply current is sensed to determine changes within the predefined periodicity. There is still the case in which the secondary has no power to operate and the primary side may assume the load is regulated. In order to prevent this case, we perform load modulation from time to time as an alive signal (just a periodic pulse), indicating that the secondary is still being powered and properly regulated. In the absence of detection of such signals, the frequency is increased towards $f_{o}$ until the primary senses the secondary again. Note that even if the secondary is working and the primary cannot sense changes in current, this is interpreted as there is no regulation.

Two microcontrollers are used in order to generate the variable switching frequency, sense the input current and sense the output voltage. It should be noted that this does not necessarily represent hardware overhead as some sort of digital processor is always required at both ends. The analog-todigital converters (ADCs) from the microcontrollers are used to sense the voltage at the load and the current variations at the primary side. The complete algorithm is partitioned into the two microcontrollers, being possible to track the power delivered at the secondary along the time of operation.

\section{IMPLEMENTATION}

A prototype for the proposed system has been implemented and tested in salt water - see Fig. 5 for the complete system representation. At the driver side, the microcontroller consists of an XMC4200 $\left(\mu \mathrm{C}_{1}\right)$, and at the load side the microcontroller 




(a)

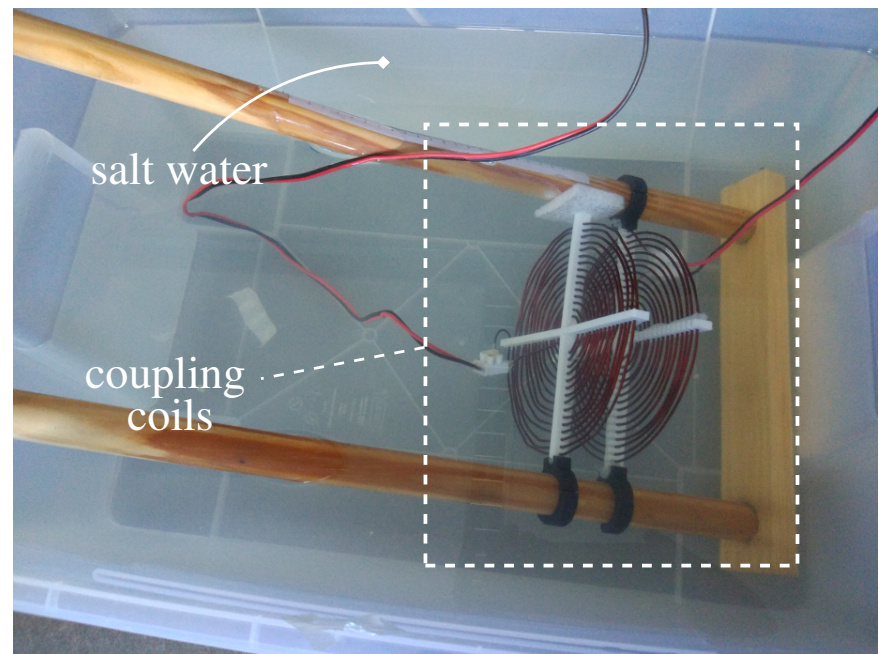

(b)

Fig. 4. (a) Test setup and (b) coupling coils in a saline water container.

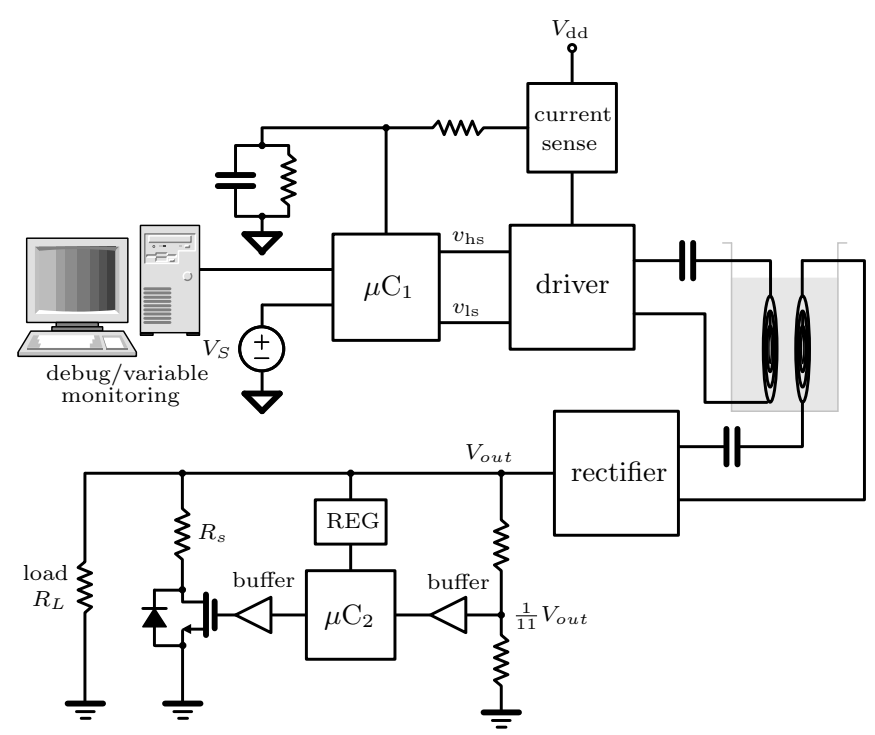

Fig. 5. Proposed adaptive system.

is an XMC1300 $\left(\mu \mathrm{C}_{2}\right)$, both from Infineon Technologies. The generated signals at the $\mu \mathrm{C}_{1}$ make use of a CCU8 timer with $80 \mathrm{MHz}$ clock frequency. The load voltage is sensed at a voltage divider (1:11) by a 12-bit ADC, whereas the reference voltage is internally defined at the $\mu \mathrm{C}_{2}$. For the power supply of $\mu \mathrm{C}_{2}$ a LM317 is used as regulator (REG), and a resistor $R_{s}$ is placed in series with the load modulation transistor to avoid the discharge of the rectifier capacitance to a level in which $\mu \mathrm{C}_{2}$ would be turned off.

The inverter in the driver employs two BSZ060NE2LS OptiMOS power MOSFETs (from Infineon Technologies), with reduced conduction resistance $\left(R_{\text {on }}<8.0 \mathrm{~m} \Omega\right)$. Load modulation is achieved by switching the power MOSFET IR LML0060TRPBF ( $R_{\text {on }} \simeq 116 \mathrm{~m} \Omega$ ) connected to ground, using a $5 \mathrm{~V}$ pulse generated by the microcontroller, buffered with a single power-supply opamp. The resistor $R_{s}$ in series with the load modulation switch, which has been defined with the value of $3.3 \Omega$, imposes the step amplitude. Besides ensuring that the $\mu \mathrm{C}_{2}$ is not powered off, it also defines the minimum current variation that can be sensed at the transmitter.

The microcontroller at the output requires a minimum voltage of $6 \mathrm{~V}$ in order to start operating. In case of power off, the lack of periodic detection of alive signals forces the driver to increase the transmitting power (i.e. $f \rightarrow f_{0}$ ). Current sensing is performed at the driver, on the high-side switching transistor, using a current shunt monitor comprised by a $0.050 \Omega$ resistor together with the difference amplifier AD8219 from Analog Devices. The signal is then applied to the 12-bit ADC of the microcontroller. Fig. 4(a) shows the test setup with driver and rectifier systems, and instrumentation; Fig. 4(b) shows the coupling coils immersed in saline water $(2 \mathrm{~g} /$ liter of salt). A spiral geometry with 15 turns has been adopted targeting low conduction losses in the water medium. The distance between coils is $4.0 \mathrm{~cm}(16 \mathrm{~cm}$ outer diameter) and the coupling factor as well as $L$ parameter were indirectly obtained from scattering parameter measurements, being their values $\simeq 0.30$ and $\simeq 18.25 \mu \mathrm{H}$, respectively [13].

The algorithm was implemented in Digital Application Virtual Engineer (DAVE ${ }^{\mathrm{TM}}$ ), i.e. the development platform for $\mathrm{XMC}^{\mathrm{TM}}$, which is an Eclipse-based integrated development environment (IDE). The $\mu \mathrm{C} /$ Probe $^{\mathrm{TM}} \mathrm{XMC}^{\mathrm{TM}}$ developed by Micrium, which allows to read and write the memory of the microcontroller during run-time and visualize the acquired data, was used along with $\mathrm{DAVE}^{\mathrm{TM}}$. This is extremely helpful during implementation and debug of the algorithm. For instance, during the load regulation procedure, the switching frequency provided at the driver (synthesized by the microcontroller) is tracked to see how the regulation is taking place (see the plot in Fig. 6).

Table I summarizes the measurement results of the output regulated voltage, $e_{V_{\text {out }}}$, and efficiency, $\eta$, for extreme and 


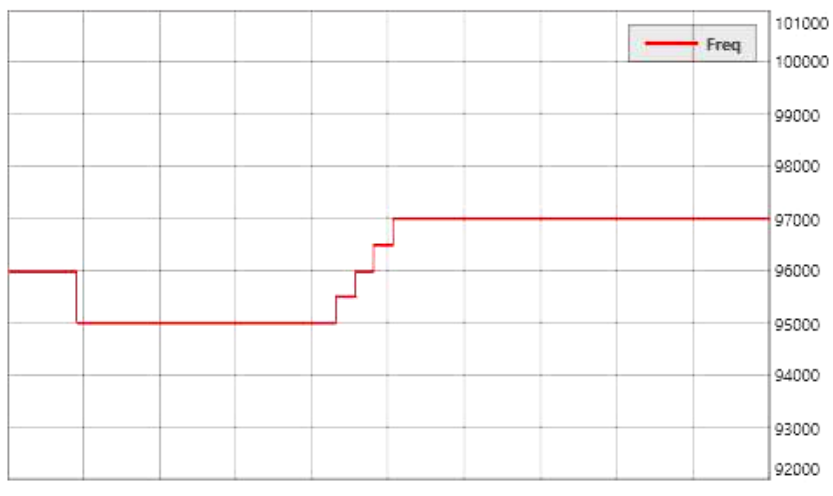

Fig. 6. Frequency variable monitored in the driver-side microcontroller during output voltage regulation ( $x$-axis represents time with 20 sec/tick).

TABLE I

Voltage REgUlation RESUlts WITH DEVIATIONS ON $V_{\text {DD }}$ AND $R_{L}$.

\begin{tabular}{cccccc}
\hline $\begin{array}{c}R_{L} \\
(\Omega)\end{array}$ & $\begin{array}{c}V_{\mathrm{dd}} \\
(\mathrm{V})\end{array}$ & $\begin{array}{c}e_{V_{\text {out }}} \\
(\%)\end{array}$ & $\begin{array}{c}\Delta f_{\text {tune }} \\
(\mathrm{kHz})\end{array}$ & $\begin{array}{c}P_{\text {in }} \\
(\mathrm{W})\end{array}$ & $\begin{array}{c}\eta \\
(\%)\end{array}$ \\
\hline & 6.0 & 0.1 & 0.0 & 3.60 & 60.7 \\
25.8 & 8.0 & 4.0 & -4.0 & 3.84 & 61.4 \\
& 10.0 & 4.9 & -6.0 & 3.87 & 62.1 \\
\hline \multirow{3}{*}{30.8} & 6.0 & 6.4 & 0.0 & 3.76 & 55.0 \\
& 8.0 & 7.2 & $-5,0$ & 3.63 & 57.9 \\
& 10.0 & 1.3 & -7.0 & 3.13 & 59.9 \\
\hline \multirow{3}{*}{37.1} & 6.0 & 6.0 & -3.0 & 3.24 & 52.6 \\
& 10.0 & 3.7 & -5.5 & 3.09 & 52.7 \\
& 10.9 & -7.2 & 3.20 & 53.1 \\
\hline
\end{tabular}

central cases of supply voltage, $V_{d d}$, and load ranges, $R_{L}$. The regulation error is measured according to $V_{r e f}=7.5 \mathrm{~V}$ (target voltage) and the tune deviation $\Delta f_{\text {tune }}$ is obtained based in $f_{o}=100 \mathrm{kHz}$. The efficiency comprises all the system losses, except for the microcontroller at the driver side, which is powered by a different power supply for debugging purposes. Fig. 7a) depicts the case when the output voltage is superior to $V_{\text {ref }}$. Hence, two pulses are generated by the load modulation circuit (signal in yellow) and are detected by the current-sense circuitry in the driver (signal in blue). This process denotes some noisy behavior essentially due to the switching action of the class-D driver. In a different time scale, Fig. 7b) shows five iterations in the voltage regulation process at the load, ending at an output voltage close to $V_{r e f}$. The periodic abrupt voltage changes along the first iterations are due to load modulation, whereas the last ones, when the voltage is already close to $V_{r e f}$, are due to alive signals with lower frequency.

\section{CONCLUSION}

This work presented a new approach in which the output voltage is regulated against load and power supply variations at the receiving and transmitting side, respectively. Resorting to periodic load short-circuits (i.e. load modulation at the secondary side), the end side is capable of communicating with the transmitter without the need for additional wireless links. Both the primary and secondary side implement simple algorithms to ensure proper operation of the WPT system. This allows for a reduced system complexity in the wireless system

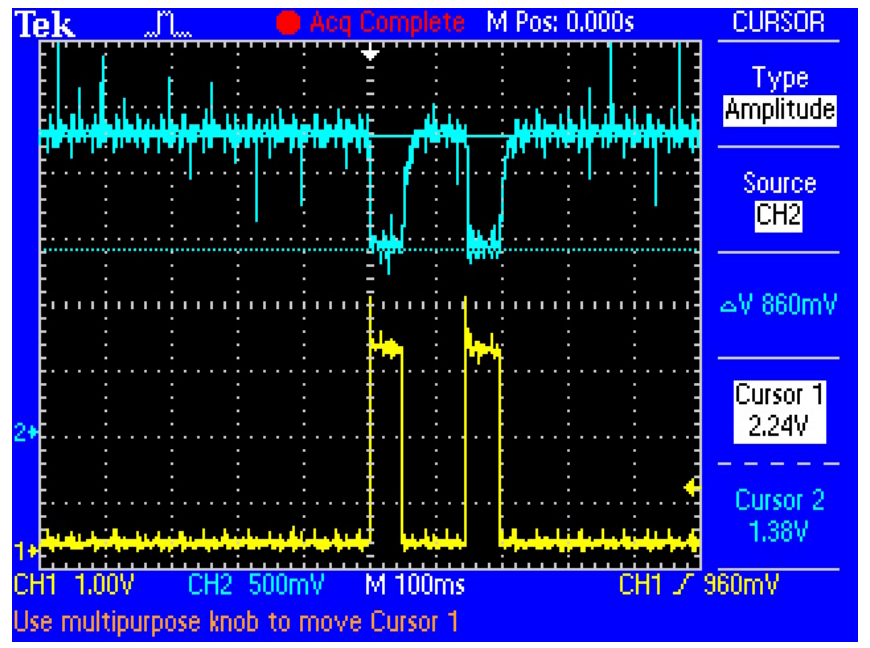

(a)

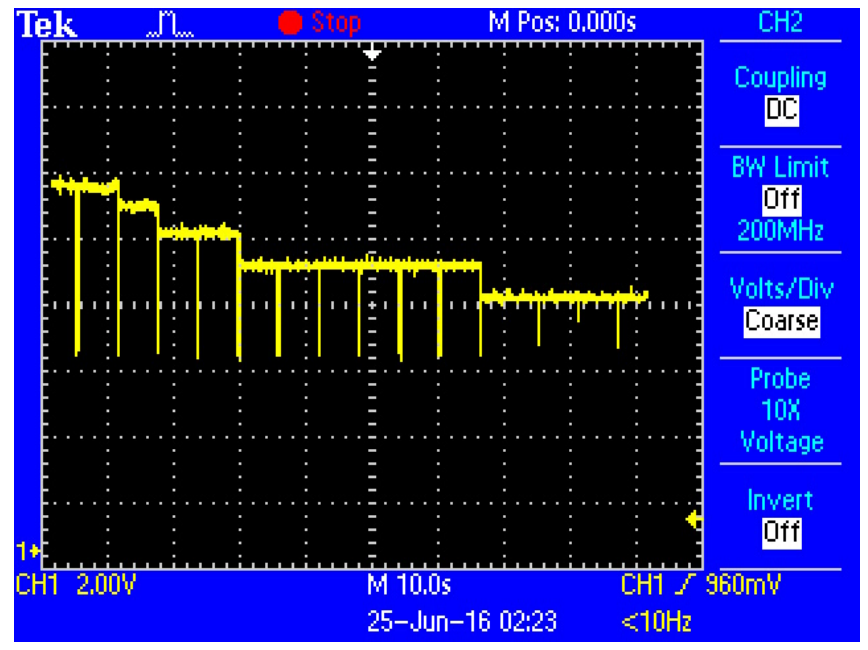

(b)

Fig. 7. (a) Pulse sense and (b) load regulation.

since only one variable is adjusted (switching frequency of the class-D driver). By adopting this methodology, a maximum regulated output voltage error of $e_{V_{\text {out }}}=7.2 \%$ is attained considering a load variation of 30 to $37 \Omega$ and supply voltage range of 6 to $10 \mathrm{~V}$. This approach still presents some limitations, namely the existing switching node noise that can introduce false positives in the algorithm and the fixed value of the load modulation resistor, $R_{s}$, that sets the effective load range in which the system is capable of performing voltage regulation.

\section{ACKNOWLEDGMENT}

This work was supported by integrated project CORAL - "NORTE-01-0145-FEDER-000036". Project "NORTE-010145-FEDER-000036" is financed by the North Portugal Regional Operational Programme (NORTE 2020), under the PORTUGAL 2020 Partnership Agreement, and through the European Regional Development Fund (ERDF). The work was also carried out in the scope of project ENDURE - 
Enabling Long-Term Deployments of Underwater Robotic Platforms in Remote Oceanic Locations (PT02 Aviso4 0015). ENDURE benefits from a $218,157 €$ grant from Iceland, Liechtenstein and Norway through the EEA Grants. The support of STRONGMAR: STRengthening MARritime technology Research Center (H2020-TWINN-2015 (CSA)-692427), TEC4SEA research infrastructure (www.tec4sea.com), and COST action IC1301 - WIPE is acknowledged.

The authors would like to thank Pedro Alves, Carlos Graf, and Vítor Pinto from DEEC/FEUP for all the help with soldering, troubleshooting, and PCB designs.

\section{REFERENCES}

[1] S. Y. Ron Hui, "Magnetic resonance for wireless power transfer [a look back]," IEEE Power Electronics Magazine, vol. 3, no. 1, pp. 14-31, Mar 2016.

[2] K. Y. Kim, C. Yoon, N. Y. Kim, J. Choi, Y. H. Ryu, D. Z. Kim, K. S. Song, C. H. Ahn, E. Park, Y. K. Park, and S. Kwon, "Magnetic resonance wireless power transfer system for practical mid-range distance powering scenario references," in IEEE International Conference on Consumer Electronics (ICCE'2013), Jan 2013, pp. 175-176.

[3] J. C. Chiao, "Batteryless wireless gastric implants," in IEEE 15th Annual Wireless and Microwave Technology Conference (WAMICON'2014), Jun 2014, pp. 1-4.

[4] $\mathrm{S}$. Li and C. C. Mi, "Wireless power transfer for electric vehicle applications," IEEE Journal of Emerging and Selected Topics in Power Electronics, vol. 3, no. 1, pp. 4-17, Mar 2015.

[5] C.-S. Wang, O. H. Stielau, and G. A. Covic, "Design considerations for a contactless electric vehicle battery charger," IEEE Trans. Ind. Electron., vol. 52, no. 5, pp. 1308-1314, Oct 2005 .

[6] J. Oiler, G. Anderson, V. Bana, A. Phipps, M. Kerber, and J. D. Rockway, "Thermal and biofouling effects on underwater wireless power transfer," in IEEE Wireless Power Transfer Conference (WPTC'2015), May 2015, pp. $1-4$.

[7] J. U. W. Hsu, A. P. Hu, and A. Swain, "A wireless power pickup based on directional tuning control of magnetic amplifier," IEEE Trans. Ind. Electron., vol. 56, no. 7, pp. 2771-2781, Jul 2009.

[8] Y. Cao, Z. Dang, J. A. A. Qahouq, and E. Phillips, "Dynamic efficiency tracking controller for reconfigurable four-coil wireless power transfer system," in IEEE Applied Power Electronics Conference and Exposition (APEC'2016), Mar 2016, pp. 3684-3689.

[9] H. Li, J. Li, K. Wang, W. Chen, and X. Yang, "A maximum efficiency point tracking control scheme for wireless power transfer systems using magnetic resonant coupling," IEEE Trans. Power Electron., vol. 30, no. 7, pp. 3998-4008, Jul 2015.

[10] T. D. Yeo, D. Kwon, S. T. Khang, and J. W. Yu, "Design of maximum efficiency tracking control scheme for closed loop wireless power charging system employing series resonant tank," IEEE Trans. Power Electron., vol. PP, no. 99, pp. 1-1, To be published 2016.

[11] A. Askari, R. Stark, J. Curran, D. Rule, and K. Lin, "Underwater wireless power transfer," in IEEE Wireless Power Transfer Conference (WPTC'2016), May 2015, pp. 1-4.

[12] P. Amaral, C. Duarte, and P. Costa, "On the impact of timer resolution in the efficiency optimization of synchronous buck converters," International Journal of Power Electronics and Drive Systems, vol. 6, no. 4, pp. 693-702, Dec 2015.

[13] F. Gonçalves, C. Duarte, and L. M. Pessoa, "A novel circuit topology for underwater wireless power transfer," in International Conference on Systems Informatics, Modelling and Simulation, Riga, Latvia, Jun 2016, pp. 181-186. 